\title{
Simulating New Drop Test Vehicles and Test Techniques for the Orion CEV Parachute Assembly System
}

\author{
Aaron L. Morris ${ }^{1}$ \\ Barrios Technology, Houston, TX, 77598 \\ Usbaldo Fraire Jr. ${ }^{2}$, Kristin J. Bledsoe ${ }^{3}$, Eric Ray ${ }^{4}$ Jim W. Moore ${ }^{5}$, Leah M. Olson ${ }^{6}$ \\ Jacobs Engineering, Houston, TX, 77598
}

\begin{abstract}
The Crew Exploration Vehicle Parachute Assembly System (CPAS) project is engaged in a multi-year design and test campaign to qualify a parachute recovery system for human use on the Orion Spacecraft. Test and simulation techniques have evolved concurrently to keep up with the demands of a challenging and complex system. The primary simulations used for preflight predictions and post-test data reconstructions are Decelerator System Simulation (DSS), Decelerator System Simulation Application (DSSA), and Drop Test Vehicle Simulation (DTV-SIM). The goal of this paper is to provide a roadmap to future programs on the test technique challenges and obstacles involved in executing a large-scale, multi-year parachute test program. A focus on flight simulation modeling and correlation to test techniques executed to obtain parachute performance parameters are presented.
\end{abstract}

\section{Nomenclature}

$\begin{array}{ll}\text { 2-DOF } & \text { two-degree-of-freedom } \\ \text { 6-DOF } & \text { six-degree-of-freedom } \\ \text { AS } & \text { Airborne Systems } \\ \text { BEA } & =\text { Best Estimate Atmosphere } \\ \text { BET } & =\text { Best Estimate Trajectory } \\ \text { BEW } & =\text { Best Estimate Winds } \\ \text { CAPSIM } & =\text { Capsule Simulation } \\ \text { CDT } & =\text { Cluster Development Test } \\ \text { CEV } & =\text { Crew Exploration Vehicle } \\ \text { CFD } & =\text { Computational Fluid Dynamics } \\ \text { CMS } & =\text { Cradle Monorail System } \\ \text { CPAS } & =\text { Crew Exploration Vehicle Parachute Assembly System } \\ \text { CPSS } & =\text { Cradle Platform Separation System } \\ \text { DSS } & =\text { Decelerator System Simulation } \\ \text { DSSA } & =\text { Decelerator Simulation System Application } \\ \text { DTV } & =\text { Drop Test Vehicle } \\ \text { DTV-SIM } & =\text { Drop Test Vehicle Simulation } \\ \text { DZ } & =\text { Drop Zone } \\ \text { EFTC } & =\text { Energy Force Transfer Coupling } \\ \text { EIT } & =\text { Engineering Investigation Team } \\ \text { ESCG } & =\text { Engineering Services Contract Group } \\ \text { FDP } & =\text { Flight Data Processor } \\ \text { Gen } & =\text { Generation } \\ & \end{array}$

${ }^{1}$ CPAS Analysis IPT Chair, Aerothermal and Flight Mechanics, 455 E. Medical Center Blvd., Webster, TX.

${ }^{2}$ CPAS Analysis Engineer, Aerothermal and Flight Mechanics, 455 E. Medical Center Blvd., Webster, TX.

${ }^{3}$ CPAS Analysis Engineer, Aerothermal and Flight Mechanics, 455 E. Medical Center Blvd., Webster, TX.

${ }^{4}$ CPAS Analysis Engineer, Aerothermal and Flight Mechanics, 455 E. Medical Center Blvd., Webster, TX.

${ }^{5}$ CPAS Analysis Engineer, Aerothermal and Flight Mechanics, 455 E. Medical Center Blvd., Webster, TX.

${ }^{6}$ CPAS Analysis Engineer, Aerothermal and Flight Mechanics, 455 E. Medical Center Blvd., Webster, TX. 


$\begin{array}{ll}\text { GUI } & =\text { Graphical User Interface } \\ \text { JDTV } & =\text { Jumbo Drop Test Vehicle } \\ \text { LVAD } & =\text { Low Velocity Airdrop Delivery } \\ \text { MDS } & =\text { Mid-Air Delivery System } \\ \text { MDTV } & =\text { Medium Drop Test Vehicle } \\ \text { PCDTV } & =\text { Parachute Compartment Drop Test Vehicle } \\ \text { PTV } & =\text { Parachute Test Vehicle } \\ \text { PTV2 } & =\text { Parachute Test Vehicle } 2 \\ \text { SRB } & =\text { Solid Rocket Booster } \\ \text { YPG } & =\text { Yuma Proving Ground }\end{array}$

\section{Introduction}

$\mathrm{T}$ HE Crew Exploration Vehicle (CEV) Parachute Assembly System (CPAS) must be tested at realistic conditions to ensure the system's performance is robust and reliable during all possible deployment conditions. Critical flight conditions are defined by the CPAS Drogue and Main test envelope ${ }^{1}$. Key factors that affect parachute performance are aerodynamic wakes and atmospheric conditions at altitude. The aerodynamic wake created by a capsule shaped vehicle requires investigation to attain a better understanding of inflation performance effects. Similarly, high dynamic pressure conditions are necessary to investigate to understand inflation performance when higher velocity, extreme atmospheric and orientation cases are experienced.

The development of new test vehicles and increasingly complex test techniques was necessary to attain a representative deployment condition at higher altitudes and verify performance requirements. Logistical issues including aircraft availability, altitude capability, and size constraints led to the design of several test vehicles with various configurations used to satisfy specific CPAS Drogue deployment test conditions. Concessions and constraints were faced during the development of these vehicles. Not all atmospheric or aerodynamic conditions could be tested using a single vehicle or test technique during the first (Gen I) generation of testing. Certain test techniques delivered results for a single test condition, but were vital to the development of increasingly complex and representative test methods necessary for the next generation of testing.

Similar to the test technique advancements, simulation and analysis techniques used to model vehicle and parachute dynamics also experienced an evolution to meet analysis demands. Initial preflight predictions and data reconstructions for Gen I were based on low fidelity calculations and founded on simplified vehicle and parachute models. The completion of the Gen I test phase resulted in a need for higher fidelity simulations to predict test performance for Gen II. As more test data was acquired, an improved understanding of parachute performance parameters was achieved. Flaws, omissions, and limitations in the simulation and analysis techniques were identified through perpetual application which were required to provide test support. As a result, the identified limitations have led to an evolution of new simulation and analysis techniques that are required to understand dynamic extraction, separation, and inflation test events for supporting the Gen III testing phase.

\section{Evolution of CPAS Test Vehicles and Techniques}

Heritage test vehicle designs and techniques were utilized at the inception of Gen I for testing single and cluster parachute configurations. Single Pilot, Drogue, and Main parachute architectures were tested initially to characterize inflation parameters. Once the inflation characteristics of each individual parachute were determined, CPAS moved on to the Cluster Development Test (CDT) series to demonstrate the system. A weight tub on a Type V platform was used to test clusters of parachutes and a Medium Drop Test Vehicle (MDTV) was used to reach a higher dynamic pressure test condition. Initial Gen I tests were used as an opportunity to test the instrumentation and data acquisition system and therefore only required release altitudes under 14,000 ft. The parent aircraft used for these tests were HH-1, UH-1 or CH-47 helicopters and C-130A aircraft. As higher release altitudes became necessary, a cradle structure was needed to deploy a Medium Drop Test Vehicle (MDTV) from the cargo area of an aircraft. A Cradle Monorail System (CMS) was designed and developed to serve this purpose. An MDTV/CMS integrated vehicle allowed release altitude capabilities to reach $25,000 \mathrm{ft}$ on a C-130A. Low Velocity Aerial Delivery (LVAD) techniques were also used to extract various Type V platform test configurations from a C-130A. Variations of weight tub and Type $\mathrm{V}$ platform vehicle configurations were developed to satisfy evolving test requirements. A Parachute Test Vehicle (PTV) was used at the end of Gen I to demonstrate the system's performance using a representative capsule. An LVAD extraction technique was executed for CDT-2 to satisfy aircraft and release 
altitude requirements. The mentioned vehicles were the primary test articles used for the first (Gen I) and second (Gen II) generation of testing.

Each designed vehicle fulfill a specific test objective. An example is a Medium Drop Test Vehicle (MDTV). This vehicle delivered a high dynamic pressure condition. A second example is a Parachute Test Vehicle (PTV). This vehicle fulfilled a different purpose by providing a similar wake to an Orion capsule. By designing multiple test vehicles, CPAS experienced a large range of challenges which in turn allowed for a growth in knowledge of each test technique's implementation. Not only has CPAS designed multiple test vehicles, it has also completed two generations of testing, Gen I and Gen II, with a third generation (Gen III) starting in the near

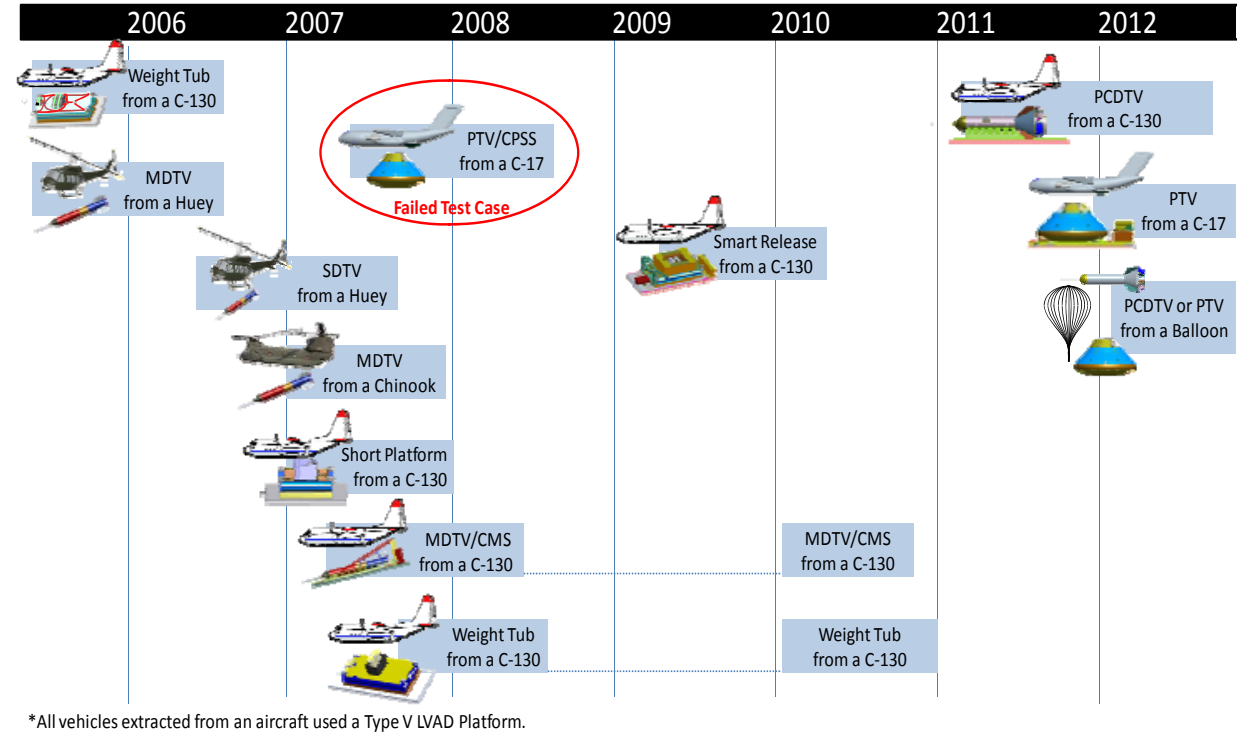

Figure 1: Evolution of CPAS Test Vehicles and Techniques

future. Each generation of testing builds off the lessons learned from the previous. The evolution of CPAS test vehicle and techniques are summarized on a timeline in Figure 1.

As the project evolves into the third (Gen III) generation, new vehicles are being developed such as the Parachute Compartment Drop Test Vehicle (PCDTV) and PTV-2, which have evolved from predecessor vehicles such as the MDTV and PTV. The Gen III vehicles are intended to test representative systems and incorporate the lessons learned from the Gen I and Gen II phases to meet the evolving testing needs of CPAS. Similar to the Gen II CMS, the Mid-Air Release System (MDS) is a Gen III cradle designed to accommodate a PCDTV. Other test techniques discussed include a delayed load transfer platform used for extraction and a mid-air separation technique of an Type V platform.

Testing failures and challenges experienced with the aerodynamic design, flight mechanics, and implementation of the various vehicle designs are included to provide insight on why new testing and simulation techniques were developed. The test malfunction experienced on CDT-2 helped the team identify alternative test methods and the development of a smart release algorithm based on orientation logic.

\section{Evolution of CPAS Simulation and Analysis Techniques}

At the inception of Gen I, the primary objective was to demonstrate performance capabilities of CPAS parachutes and determine a baseline design through test and analysis. A second parallel objective was to attain a general understanding of parachute inflation parameters, rate of descent (ROD), and loads experienced by the system. Two-degree-of-freedom (2-DOF) analyses were performed using low fidelity spreadsheet calculations to extract inflation characteristics that include the over-inflation constant, effective reefing ratio, canopy fill constant, opening profile shape exponent, parachute fill time, and drag area. Early simulation and analysis techniques delivered a rough order of magnitude of performance results. Analysis demands required the development of higher fidelity simulations to provide real-time preflight predictions and more accurate post-flight data reconstructions. Legacy analysis methods were no longer applicable to support CPAS mission operations. New trajectory simulations were introduced and analysis techniques became increasingly complex. Monte Carlo capabilities were not available during Gen I and were introduced for all CPAS simulations during Gen II. This analysis advancement allowed statistical analysis of thousands of test cases to be performed in a matter of hours versus days ${ }^{2}$. This section will expand on the evolution of CPAS simulation and analysis techniques from Gen I and Gen II. Planned Gen III 
simulation enhancements and model developments are also included. A timeline summary of each simulation used for preflight predictions, mission support, and post-flight reconstructions are shown in Figure 2. Newer versions of each simulation have been released since 2006 to evolve with model advancements and inflation understanding.

\begin{tabular}{|c|c|c|c|c|c|c|}
\hline Time & 2006 & 2007 & 2008 & 2009 & 2010 & 2011 \\
\hline \multirow{8}{*}{ Preflight Predictions } & 2-DOF Spreadsheets & & & & & \multirow{3}{*}{ V.Beta__8ge } \\
\hline & DSSA vBeta_5a, vBeat_5b4, vBeta__5b5 & vaBeta_be & v.Beta_ffil & v.Beta_8f3, vBeta__8f5 & |v.Beta_8g, v.Beta__ggl & \\
\hline & \multirow{6}{*}{ DTV-Sim v.14 } & & & & Monte Carlo capability & \\
\hline & & & v.15 & & & \multirow[t]{2}{*}{ v.17 } \\
\hline & & & & \multirow[b]{4}{*}{ More Cabb caphitity } & \multirow[t]{4}{*}{ Monte Carlo capability } & \\
\hline & & & Pallet Sim & & & \multirow{3}{*}{ Separation Simulation } \\
\hline & & & DSS & & & \\
\hline & & & & & & \\
\hline \multirow{3}{*}{ Mission Support } & X-38 Legacy Footprint Tool & (Primary) & (Primary) & (Primary) & & \\
\hline & & & & Sasquatch (Secondary) & (Primary) & \\
\hline & & & & & Pressure Altitudele Calculation & \\
\hline \multirow{6}{*}{$\begin{array}{l}\text { Post Flight } \\
\text { Reconstruction }\end{array}$} & 2-DOF Spreadsheets & & & & & \\
\hline & \multirow{5}{*}{ DTV-Simv14 } & DSSAvB:eta_ze & |vBEta_ffil & & & \\
\hline & & & v.15 & & & \\
\hline & & & DSS & & & \\
\hline & & FDP v1.1.07 & & v1.1.09, vi.1.10 & & \\
\hline & & & & BET/BEA/BEW Scripts & & \\
\hline
\end{tabular}

Figure 2: Evolution of CPAS Simulation and Analysis Techniques

\section{A. Trajectory Simulations}

The simulations used for supporting CPAS analysis efforts evolved from 2-DOFs and spreadsheet calculations in Gen I to six-degree-of-freedom (6-DOF) Monte Carlo simulations that have an increased capability of delivering analysis of representative test architectures. Each simulation has limitations and is used for specific analysis tasks. New analysis techniques have been developed to mitigate these identified limitations with the intent to deliver a representative trajectory model required for preflight test efforts. Analysis techniques have and are being developed to fill modeling gaps in the simulations. Multiple simulations are also used concurrently in some cases to provide a complete trajectory analysis. All the simulations and techniques used by CPAS are included in the subsequent sections.

1) DSSA

The Decelerator Simulation System Application (DSSA) ${ }^{3}$ is a 6-DOF simulation based on the UD-233A legacy simulation. This simulation tool is used to provide end-to-end flight predictions of Type V LVAD test configurations such as a weight tub. Altitude contingency or mass property studies can be performed using DSSA and is valuable when determining preflight release altitudes based on atmospheric conditions. The simulation uses an Excel Graphical User Interface (GUI) front end as shown in Figure 3 to execute its Fortran executable. The GUI provides input options within various tabs to develop a simulation that represents the test configuration. A useful tool within DSSA is its extraction model, which is used concurrently with other simulations for the initial extraction flight phase from a fixedwing aircraft. The simulation can be initiated using the extraction

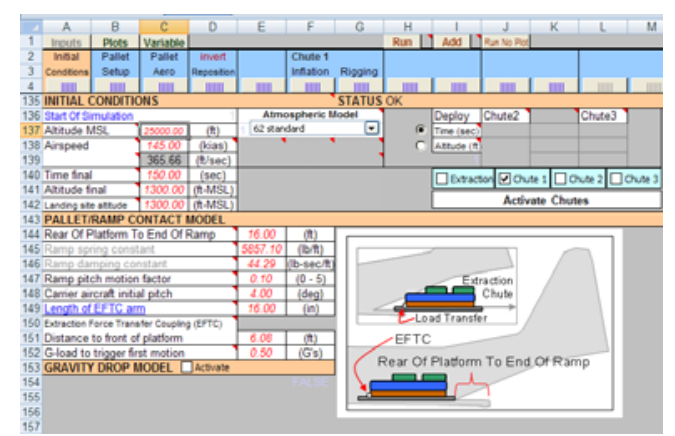
parachute model and has options to activate three subsequent parachutes in series with specific release altitudes, cut times, inflation, rigging, and atmospheric model input selections. Maintenance of DSSA has been ongoing and required to improve the fidelity of the simulation. The latest released version of DSSA was Beta8g2 and included an update to all inflation models including added mass ${ }^{4}$. The introduction of DSSA in Gen I was the start of the advancement of simulation tools that can be employed to deliver higher fidelity predictions and was specifically successful for predicting pallet type vehicle performance.

2) DTV-SIM

The Drop Test Vehicle Simulation (DTV-SIM) is a 2-DOF simulation introduced in the early phase of Gen I to provide trajectory analysis for any type of vehicle body including dart shaped vehicles from a specified release altitude. The simulation was a leap forward from 2-DOF spreadsheet calculations and allowed the analysis engineer 


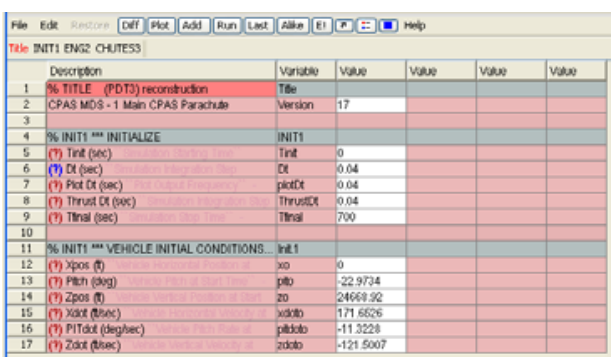

Figure 4. DTV-SIM GUI

to input initial vehicle conditions, mass properties, cutter times, and inflation parameters for multiple reefed stages. The simulation did not include an extraction model and assumed the vehicle to be a point mass. The simulation was initially developed for providing trajectory analysis for Solid Rocket Booster (SRB) parachute tests and adopted by CPAS for delivering MDTV and CMS trajectory predictions. Several simulation flags used for SRB trajectory analysis such as engine system inputs were deactivated because they were not applicable to CPAS test architectures. Like DSSA, DTV-SIM has the capability to deliver contingency studies based on altitude, mass properties, or drag area. The simulation was sufficient for providing preflight predictions of MDTV drop tests from helicopters at altitudes under 14,000 ft. The key limitation for DTVSIM was the lack of an extraction model and could not be the sole simulation used for predicting performance from an aircraft. The latest version of DTV-SIM currently used for initial 2-DOF analysis is version 17. The simulation is developed using Matlab scripts that represent various models. The primary models that make up the simulation include load, drag area, atmospheric, aero, and trajectory files. Matrix files are also included in the simulation to capture and organize data used for delivering final output results. DTV-SIM is a lower fidelity tool and is used to deliver initial trajectory analysis for test architectures and new dart shaped vehicle designs such as the PCDTV. Due to the simulation assuming a point mass, DTV-SIM is also utilized to deliver trajectory analysis of the CMS or MDS cradle structures.

\section{3) DSSA2DTV-SIM Analysis Techniques}

For test architectures involving a two-vehicle system such as the MDTV/CMS test technique, DSSA and DTVSIM had to be used concurrently to deliver a representative trajectory. DSSA was executed to obtain end conditions from the aircraft extraction model using an integrated vehicle configuration. The simulation was executed for typically the first 10 seconds of the flight to determine when the lowest dynamic pressure condition was reached. The end conditions obtained from the DSSA extraction model were then applied as initial conditions for DTV-SIM. This simulation technique assumed an instantaneous separation event of the MDTV from the CMS. Earlier Gen I techniques used the Excel output obtained after running a case in DSSA to determine initial conditions for DTVSIM. A new simulation technique was developed for Gen II and DTV-SIM is now automated to obtain end conditions from DSSA with the development of Matlab scripts used to allow the simulation to read data files from DSSAs extraction Model. The enhancement of DTV-SIM reduces human error in the calculation of initial conditions. DSSA is the only simulation used by CPAS that has an extraction model. Similar extraction modeling techniques are used with other simulation tools such as DSS, CAPSIM, and PalletSIM.

4) DSS

Decelerator System

Simulation (DSS) is a higher

fidelity 6-DOF analysis tool

that was introduced and

employed by CPAS at the end of Gen I. DSS was previously used by the X-38 program and is also a legacy simulation of UD-233A. DSS has increased capabilities for simulating multiple aspects of a drop test including payload forces, dynamic pitching motions, and payload trajectory during the entire descent phase ${ }^{5}$. The simulation is more complex to execute compared to DSSA and DTV-SIM and can be used to evaluate test vehicle concepts or predict test performance. Representative

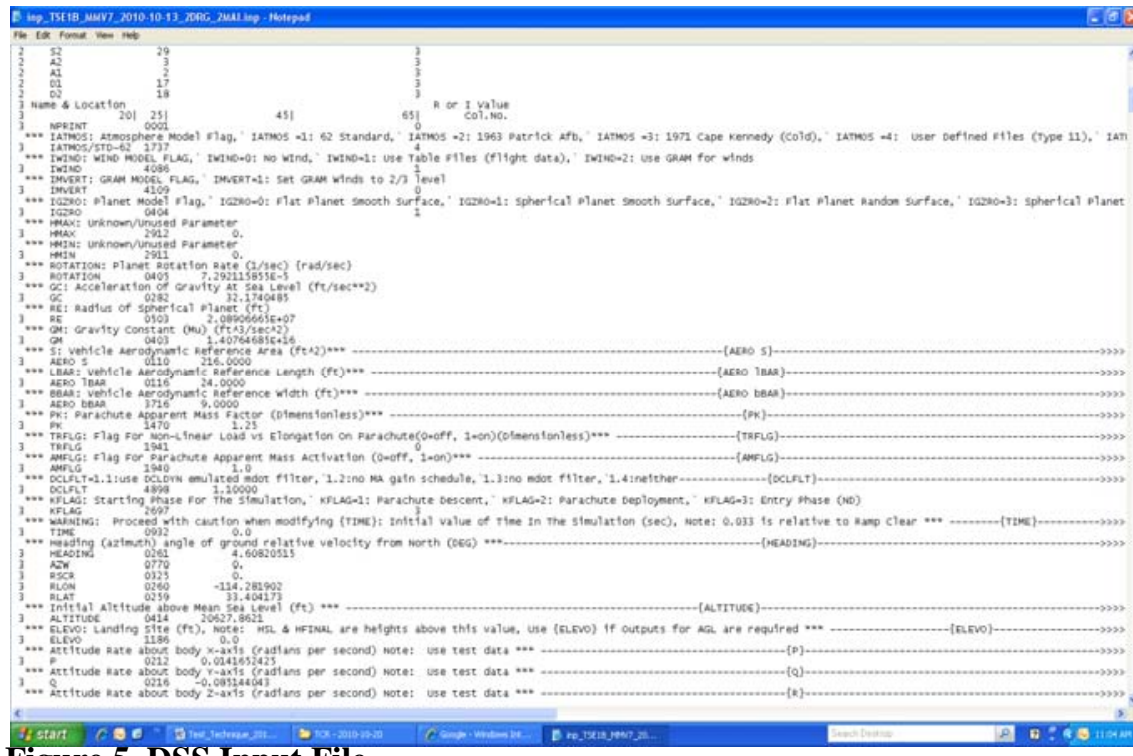

Figure 5. DSS Input File

aerodynamic input files are need to characterize specific vehicle configurations and atmospheric data files such as 
density and wind velocities are necessary to simulate environment conditions for each altitude phase. Mass properties and dimensions of the test vehicle configuration are also vital to developing a representative trajectory. The simulation uses a composite model approach to simulate parachute clusters and a single parachute modeling technique has not been performed using DSS. Model limitations employed by DSS include the riser-harness spring and damper constants, confluence, and added mass. These models require maintenance and revision to improve the fidelity of the simulation results. A minor DSS limitation relates to the simulation deployment methods for Pilot and Drogue parachutes. Each is mortar deployed and no direct simulation option is available for static-line deploying parachutes in the deployment sequence. In order to simulate a representative test architecture simple input file modifications can be applied to artificially static-line deploy the next parachute in the sequence. The latest Gen II version of DSS has mitigated the added mass model discrepancy by emulating the added mass model used in DCLDYN ${ }^{6}$. This solution made the added mass models used by the two simulations consistent to ensure similar prediction results are attained. DSS is currently executed via Matlab to obtain preflight and post-test data reconstruction results. A screenshot of the DSS input file is shown in Figure 5.

5) CAPSIM \& PalletSIM Simulation Technique

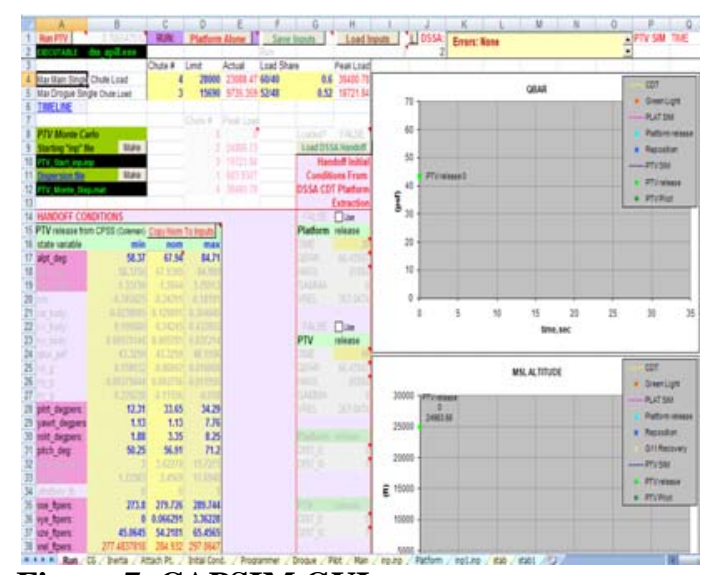

Figure 7. CAPSIM GUI

The Capsule Simulation (CAPSIM) and Pallet Simulation (PalletSIM) are variants of the legacy DSS, and were used to independently model the PTV and CPSS for the Gen I CDT-2 test, respectively. No separation simulation was developed or available for this test and therefore, a instantaneous separation was assumed. The simulation technique used to provide a complete preflight prediction included the application of DSSA, CAPSIM, and PalletSim. No individual simulation was available to provide a complete preflight prediction. DSSA provided end conditions that were manually handed over to CAPSIM and PalletSIM as initial conditions. Each respective simulation used these initial conditions to complete the

simulation from separation to touchdown. This new simulation technique provided CPAS with a method for attaining a representative preflight predictions using available simulation tools. These simulations were developed during the latter phase of Gen I and did not included the updated added mass emulator previously mentioned. The simulation was executed identical to DSS using a modified input file and shared the same limitations. Screenshots of the CAPSIM and PalletSIM are shown in Figure 7 and Figure 6, respectively.

\section{B. Post-Flight Data Reconstructions}

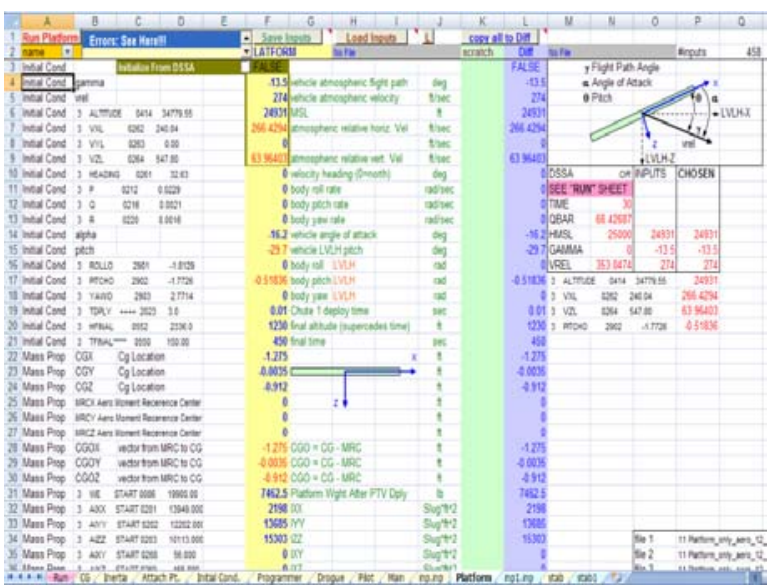

Figure 6. PalletSIM GUI

At the completion of each test, data is acquired and processed to perform data reconstructions needed to obtain parachute inflation parameters. The techniques used for completing a post-flight data reconstruction have evoled. Early Gen I reconstruction methods were comprised of plotting data on an Excel spreadsheet to determine rough performance parameters such as ROD, loads, and inflation characteristics. The introduction of DSSA and DTV-SIM during the early phases of Gen I provided an improvement to the reconstruction process but continued to be a low fidelity approach. A Flight Data Processor (FDP) was developed in response to requiring a higher fidelity reconstruction process. The Gen I FDP provided a step forward in the reconstruction process, but did have limitations. DSS became the new simulation used for reconstructing Gen II tests and has been used to date. The development of Best Estimate Trajectories (BET) was introduced to be used with DSS for a more sophisticated approach to extracting inflation parameters from test data. The following sections describe how the reconstruction process used by CPAS evolved from Gen I through Gen II. 


\section{1) Flight Data Processor (FDP)}

The latter phase of Gen I saw the development of a Flight Data Processor (FDP) that provided increased capabilities. The FDP allowed the import of flight data plus the capability to compare with preflight predictions and co-plot with simulation results. Prior to the development of the FDP, low fidelity spreadsheet calculations were the primary method for performing post-flight data reconstructions. The spreadsheet reconstruction approach involved simplified methods for plotting data and analyzing results to extract inflation parameters. The latest version of the CPAS Flight Data Processor (FDP) used was version 1.10. A screenshot of the FDP GUI is shown in Error! Reference source not found..The FDP was Matlab based and provided options that could be tailored to represent a specific test architecture. Options included selecting test grounds, tracking data type and importing atmosphere and instrumentation files. FDP was very useful only if data was delivered using consistent formats. The analysis tool lacked flexibility and required the development of new analyses methods when new or custom data was delivered. The Gen I reconstruction method had limited capabilities and required continuous maintenance to ensure accurate performance parameters were being derived.

\section{2) DSS}

Advancements in Matlab plotting techniques and data reduction methods led

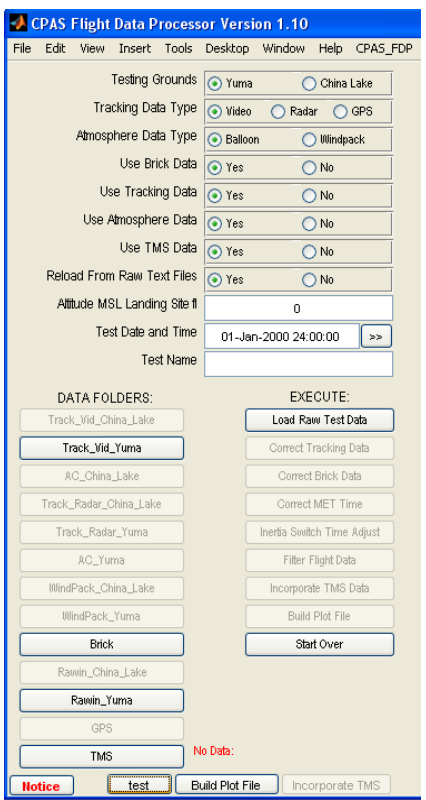

Figure 8. FDP GUI to the adoption of DSS as the primary simulation used for Gen II post-flight data reconstructions. Trajectory, winds, and atmospheric data were processed into, "Best Estimate," files, which underwent a validation process to ensure the data used was accurate prior to importing into DSS. Load and accelerometer data were used concurrently with the best estimate files and were the basis of the data reconstruction process. As mentioned in Section II.A.4, the mass properties of the vehicle are key to developing a representative reconstruction model. Engineering judgment was used to perform the first data reconstruction using DSS which resulted in the development of the, "CPAS Engineering Development Unit Operating Modeling Parameters Version 6." As more DSS reconstruction experience was attained and performance parameters were established, the seventh version of the parachute modeling memo used version six inflation parameters as an initial estimate to begin the reconstruction process. Each parameter was changed iteratively until a best fit solution of the test data was achieved. The primary objective for the data reconstruction was to match load profiles first, drag area profiles second, and use engineering judgment to determine the best overall solution. This simulation technique remains the primary method for performing data reconstructions to date.

\section{Monte Carlo Analysis}

In the beginning phases of Gen I, no automated Monte Carlo capabilities were available; only five cases could be delivered at a time and required each input to be varied manually. This was a tedious task and allowed for human error to effect final results. The development of the CAPSIM discussed in Section II.A.5 included a Monte Carlo capability and was introduced on CDT-2. This was the first automated Monte Carlo simulation used for CPAS and delivered statistical analysis of hundreds of dispersed cases. The Monte Carlo capability was only limited to CAPSIM and was not executed for PalletSIM. This simulation enhancement led to a need to develop and extend a Monte Carlo capability for all the simulations used by CPAS. A Monte Carlo for DSSA and DTV-SIM was introduced at the beginning of Gen II. This new automated analysis technique allowed preflight prediction results to account for hundreds of alternative performance scenarios; a capability that was not available at the beginning of Gen I.

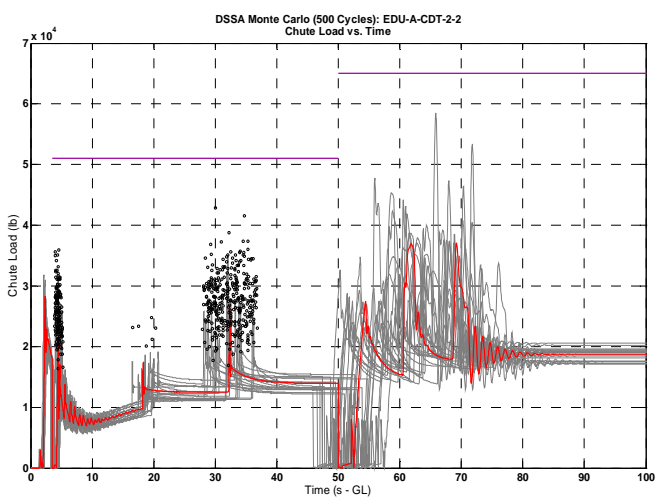

Figure 9: Monte Carlo Output was not available at the beginning of Gen 


\section{Footprint Simulations}

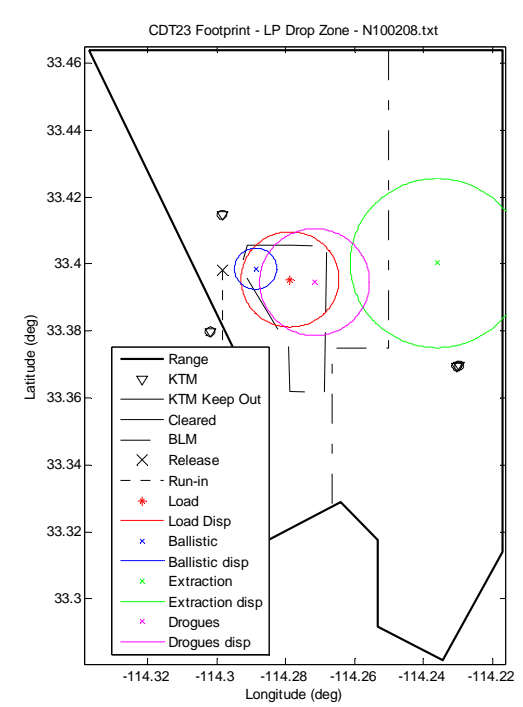

Figure 10. Sasquatch Output

A legacy X-38 Range Footprint tool, used for Gen I was the primary simulation for delivering release and landing location predictions of test articles during mission operations. The footprint simulator used an MS-DOS executable to deliver output results on a generic map used to represent a Drop Zone (DZ). The driver for the development of a new footprint analysis tool was based on the inability to modify the source code of the X-38 legacy Range Footprint tool, which limited the ability to enhance the simulation to meet CPAS needs. The new footprint analysis tool, called Sasquatch ${ }^{17}$, was tested and validated during the Gen I testing phase. This simulation tool evolved to meet the landing location predictions of test articles required by CPAS for Gen II testing. The X-38 legacy simulator was no longer used for footprint analysis and Sasquatch was adopted as the primary footprint tool at the start of Gen II. New features included the capability to import atmospheric balloon file data delivered hourly during day of flight activities. Sasquatch delivered an output solution acquired from input conditions onto a DZ map that visually provided the mission operations team with a dispersion circle around the predicted landing location of potential alternative landing locations. These results were required to ensure all DZ landing requirements were satisfied. Sasquatch had the capability of plotting various landing locations of various test articles simultaneously. The heritage footprint tool did not have this feature and required a tedious procedure to obtain the final footprint solution. Sasquatch mitigated these issues with a user friendly interface and prompts to help the user identify errors, import correct data files, and select a variety of DZ options to customize the analysis to a specific test scenario. As more tests were executed, Sasquatch was refined by obtaining actual test landing locations and using the post-flight data to improve the accuracy of the new footprint tool. A screenshot of the Sasquatch output map is shown in Figure 10.

\section{E. Simulation Advancements for Gen III Analysis}

The end of the Gen II testing phase experienced an evolution in overall simulation and analysis techniques. Data reconstruction methods were enhanced and all the simulation tools used by CPAS obtained an inflation model revision. DCLDYN's added mass model was emulated and incorporated into DSS, DSSA, and DTV-SIM. Simulation capabilities evolved to meet the simulation demands of CPAS for test support. Sasquatch was developed in response to a need of an increasingly accurate landing location simulation and Monte Carlo capabilities were extended to all the simulation tools used by CPAS. Individual simulations have either been developed or used to understand specific deployment events such as the inertial loads experienced in the risers of the system.

Each simulation has limitations and has been used to their maximum capabilities with other analysis tools to deliver representative flight predictions for various test configurations. Gen III requires testing of a representative parachute system which reflects the current Orion design. Analysis demands for Gen III include the use of sophisticated modeling techniques to ensure design limit loads are not exceeded and footprint analysis to ensure all test articles remain in the cleared DZ area. The CPAS Analysis team has made significant progress in simulation techniques and can deliver higher fidelity preflight predictions with the mentioned simulation techniques and analysis tools.

\section{1) Smart Release Algorithm}

A smart release algorithm was developed and tested during Gen II to mitigate separation issues experienced in CDT-2. The algorithm is programmed to send a signal to the cutter system when favorable orientation conditions are satisfied. If orientation conditions are met, the smart release algorithm sends the signal to release the attached vehicles and allow the separation event to occur. A back- up timer signal is available to send the cut signal to ensure the system separates if the orientation conditions are not met.

2) Separation Dynamics and Wake Effects

The development of a separation model is planned for Gen III to expand the understanding of separation dynamics. An improved understanding of aerodynamic wake effects has been attained through continual research efforts to ensure no parachute inflation malfunctions are repeated when using a capsule shaped test vehicle. The primary intent to evolve test and simulation techniques is to satisfy the rigorous requirements needed to qualify a parachute system for human spaceflight. 


\section{Gen I Helicopter Tests}

The first five tests in Gen I were executed using a Drop Test Vehicle (DTV) carried and released by a helicopter. The Pilot Development Test (PDT) series used a small DTV lifted by a UH-1. The first Drogue Development Test (DDT-1) used a Medium DTV (MDTV) lifted by a CH-47. In all of these tests, the DTV was picked up from the ground by long slings attached to the bottom of the helicopter. The helicopter flew to the desired altitude over the test range and then released the DTV. The first parachute in each testing sequence was deployed using a static line from the helicopter.

Helicopter drops were simple to execute and analyze. Preflight analysis on these tests was performed using DTV-Sim. Post-flight analyses were performed using simple spreadsheet methods.

On each Pilot test, a programmer parachute was selected and reefed to provide a specific dynamic pressure at Pilot deployment. The desired dynamic pressure increased with each test. For PDT-4, the target was 60 psf. Preflight predictions indicated that the selected programmer would provide that value; however, the actual dynamic pressure at Pilot deployment was 48.3 psf. Poor understanding of the drag of the programmer led to the failure to achieve the desired test condition.

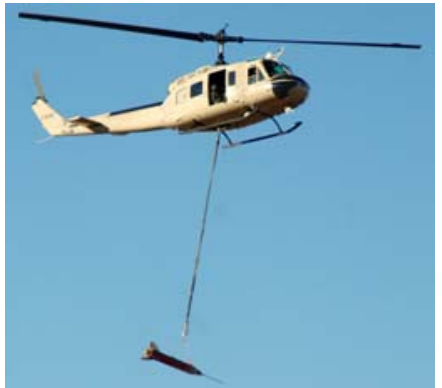

Figure 11. Small DTV Under UH-1

No programmer parachute was used on DDT-1. The Drogue parachute being tested was static-line deployed from the helicopter. An anomaly occurred during deployment when the parachute began to inflate before the riser was taut. DTV-SIM assumed parachute inflation does not begin until line stretch. In the event that additional helicopter tests were required by CPAS, the test technique and simulation would have required alteration to mitigate this anomaly. However, future tests used different test techniques that provided benefits that could not be achieved on helicopter drops.

\section{Gen I LVAD Platform with Weight Tub}

The Low Velocity Aerial Delivery (LVAD) technique allows for payloads to be parachute-extracted from a cargo aircraft at altitudes up to 25,000 ft-MSL. LVAD drops are routinely performed by the US Army and other entities, making it a well-understood and reliable technique. LVAD drops have also been used extensively on a variety of parachute development programs. Standard and modified LVAD drops form a large part of the CPAS test program to date.

For an LVAD drop, the payload is assembled on a Type $\mathrm{V}$ airdrop platform: a flat aluminum platform with rails and a restraint system. A standard LVAD payload typically consists of a weight tub of the desired weight and weight distribution, an instrumentation system, and a series of parachutes. Layers of honeycomb cardboard are stacked under the weight tub and other equipment to absorb landing impact loads. For testing, the payload is flown to the desired altitude in a cargo aircraft. A parachute deployed out of the open cargo ramp extracts the payload from the aircraft and deploys the first parachute in the test sequence.

This section discusses LVAD configurations using a weight tub as described above. Later sections will discuss other LVAD configurations used on CPAS.

\section{A. Modeling Techniques}

The Decelerator Systems Simulation Application (DSSA) was developed for the Army to model LVAD drops. For LVAD configurations with a weight tub, DSSA is used to deliver end-to-end trajectory analysis. Although DSSA has a model of aircraft extraction, the dynamics of the platform immediately after extraction remain difficult to model. The aerodynamic wakes produced by cargo aircraft are not well understood and have a significant impact on parachute and platform dynamics. In addition, winds at altitude have a strong effect on the rolling and yawing of the platform. Wind is not well-modeled in DSSA.

\section{B. Short Platform Experience}

The first CPAS Gen I LVAD test was MDT-1. A $12 \mathrm{ft}$ platform was rigged to a weight of about 6,000 lbs. Three parachutes were deployed in sequence: a $19 \mathrm{ft}$ ring slot programmer permanently reefed at 30\%, a single CPAS Pilot,

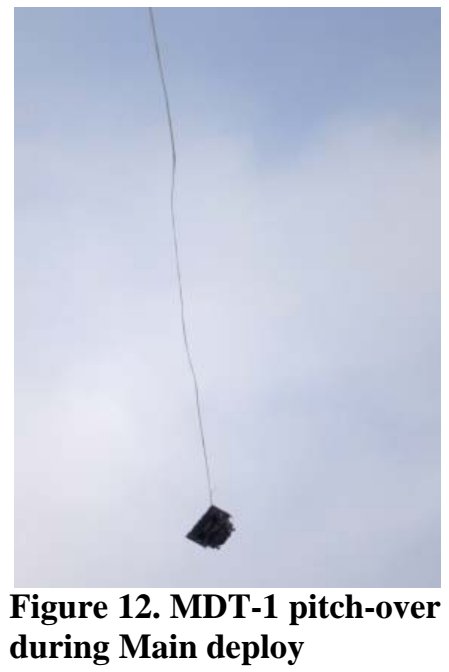


and a single CPAS Main reefed to $6 \%, 12 \%$, and full open. This test was successful, but an anomaly occurred that could have been disastrous. The platform continued to pitch significantly under the programmer. When the programmer was cut away, the platform pitched over nearly $180^{\circ}$ before the Pilot was able to deploy the Main as shown in Figure 12. A Main sling caught on a camera bracket and pulled the platform back to a bottom-down orientation. Had that not occurred, the riser or slings may have cut on the sharp edge of the platform.

The near-failure had three physical causes. The aerodynamics of small platforms are unstable and not wellunderstood. The programmer parachute was less effective than expected, in part due to the shorter-than-planned riser that was installed which put the parachute in the wake of the payload. Additionally, the reefed parachute provided a smaller drag area than the payload, preventing it from adequately controlling the platform dynamics.

MDT-2 was planned as a repeat of MDT-1. The programmer riser length was corrected, however, the platform still pitched significantly during the programmer phase. The pitch-over at programmer release was not as sharp as it was on MDT-1, but it was large enough to be a concern. Because of the results of MDT-1 and MDT-2, configurations with small platforms or programmers smaller than the payload are no longer used on CPAS tests.

\section{Aerodynamics}

LVAD platforms are, in essence, large flat plates. Flat plate aerodynamics are notoriously difficult to predict. While there are simplified calculations found in classic textbooks, these do not fully describe the complex dynamics seen on drop tests. The aerodynamics vary widely with angle of attack, which changes quickly. In simulations, it is difficult to match or predict platform motion due to aerodynamic effects.

In the early 1990s, a computational fluid dynamics (CFD) analysis was performed on an LVAD payload. The results of that study were incorporated into the simulations and used for many years as the basis for analyses. However, CFD was in its early stages at the time of that study, and the results were later found to be less accurate than was needed for high-fidelity simulations. These results were also found not to be applicable for short platforms.

The third LVAD test performed in Gen I was the first Cluster Development Test, CDT-1. In order to test the full parachute system, including the Pilot mortars, a representative parachute compartment was mounted on top of the weight tub. A wind tunnel test was conducted to determine the aerodynamic properties of the payload. The results of that wind tunnel test were incorporated into the preflight simulations to improve predictions.

A recent CFD study was used to update the previous LVAD platform aerodynamics. A variety of configurations were analyzed and found that the aerodynamic properties varied little between configurations. Therefore, because the CDT- 1 wind tunnel data was the highest-fidelity aerodynamic data available, it was chosen to be used for simulations on all subsequent tests. CFD data supplements the wind tunnel data for angles that were not tested in the wind tunnel.

\section{Trailing Distance}

The wake of a large body such as a platform or capsule can have a significant effect on the performance of a parachute. Rules of thumb are often used to determine trailing distances (the distance between the parachute and the payload). On MDT-1, a riser length of $40 \mathrm{ft}$ was selected for the programmer to ensure sufficient trailing distance. However, a $20 \mathrm{ft}$ riser was inadvertently installed instead. The shorter trailing distance brought the

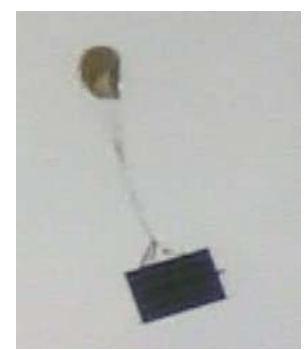

Figure 13. MDT-1 programmer buffeting programmer into the wake of the vehicle. Video analysis showed the parachute being buffeted by the wake. The open mouth area of the skirt was reduced, thus reducing the effectiveness of the parachute (Figure 13).

\section{E. Programmer Size}

As previously noted, the riser length was corrected for MDT-2, but the programmer still was not sufficient to control the vehicle dynamics. The programmer parachute on both MDT-1 and MDT-2 was a $19 \mathrm{ft}$ diameter ringslot parachute reefed to $30 \%$. Assuming a drag coefficient of 0.55 , the drag area, $\mathrm{C}_{\mathrm{D}} \mathrm{S}$, of that reefed parachute was $46.8 \mathrm{ft}^{2}$. The drag area of the 9 by $12 \mathrm{ft}$ platform was $130 \mathrm{ft}^{2}$, nearly three times the area of the parachute. In order to provide sufficient force to control a payload, a parachute must have at least the same drag area as the payload. This became a rule of thumb for future CPAS tests. 


\section{Gen I/II Missile Shaped Vehicle Drop Tests}

The MDTV was a heritage vehicle from the X-38 program and used by CPAS to reach high dynamic pressure test conditions. The CMS vehicle design was initiated by a need to develop a test technique which deployed an

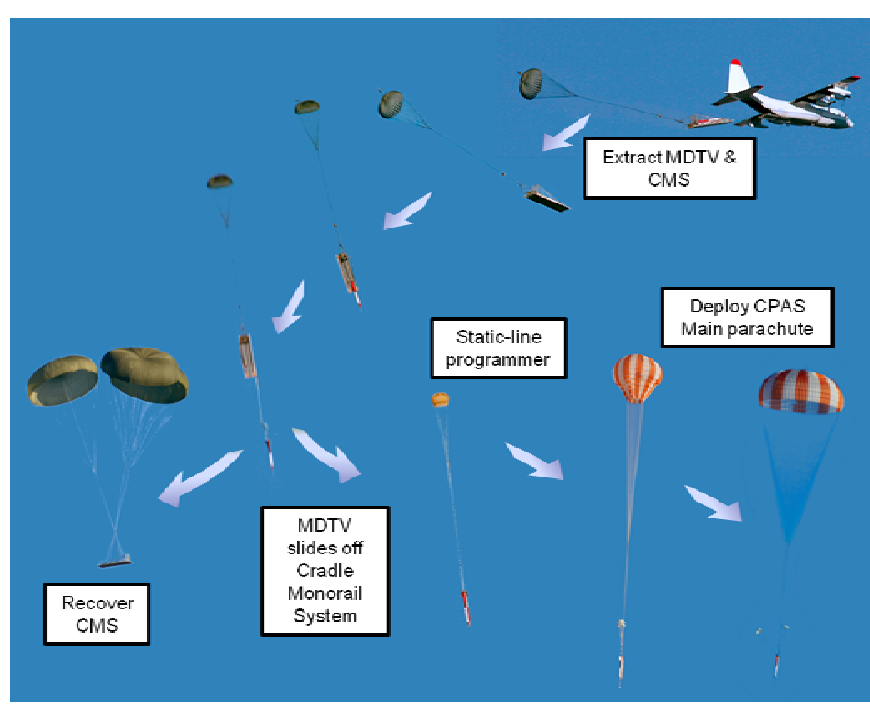

Figure 14. MDTV/CMS Test Architecture MDTV to altitudes achievable on a C-130A. Prior MDTV tests were executed using helicopters at lower altitudes as described in Section IV. Deploying an MDTV at a higher release altitudes resulted in acquiring more test data and reaching higher dynamic pressure, $\mathrm{q}_{\mathrm{bar}}$, test conditions prior to the deployment of the parachute system. The CMS was constructed on a $32 \mathrm{ft}$ Type $\mathrm{V}$ platform and used to cradle a MDTV onto the cargo area of a C-130A to ensure Army cargo requirements were satisfied ${ }^{7}$. A GFE extraction parachute rigged to an Energy Force Transfer Coupling (EFTC) mechanism was used to extract the integrated MDTV/CMS vehicle from a C130A. The EFTC was activated after ramp clear and transferred the vehicle load to the CMS backstop. An example of the MDTV/CMS test architecture is shown in Figure 14.

The simulation technique used to provide preflight predictions using DSSA end conditions as DTV-SIM initial conditions are described in Section III.A.3. No separation simulation was available for preflight predictions. As a result, other analysis methods and engineering judgment were employed to mitigate separation uncertainty and stability issues. A low dynamic pressure condition was selected using preflight prediction results from DSSA to determine a conservative time for separation events to execute. Separating at a low dynamic pressure was assumed to be the most stable moment in the flight where excitation of attitude and angular rates were minimal. The separation timeframe was dependent on the lowest $\mathrm{q}_{\mathrm{bar}}$ and selected to ensure a clean separation with minimum aerodynamic effects. Favorable $\mathrm{q}_{\mathrm{bar}}$ conditions were typically met when the integrated vehicle reached an orientation range between $30^{\circ}-90^{\circ}$ prior to the separation event. A sample $\mathrm{q}_{\mathrm{bar}}$ plot generated after running a case file in DSSA for an extracted MDTV/CMS vehicle is shown in Figure 15. The selected timeframe for

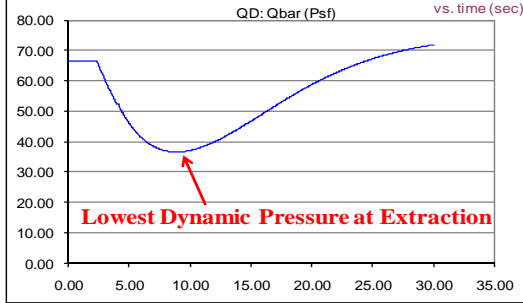

Figure 15. DSSA qbar results for extracted MDTV/CMS separation was referred to as the "bottom of the qbar bucket," and is essentially the lowest point of the concave profile.

\section{B. Separation Assumptions}

A key simulation assumption made by CPAS during Gen II included an instantaneous separation of an MDTV/CMS test technique. This initial assumption was driven by a lack of an available separation model. The simulations did not have the capability of modeling this dynamic event. Post-test video analysis corrected this assumption and proved separation events actually required 1.5 seconds for an MDTV to clear a CMS. The need for a separation model has been identified as a priority for future vehicle architectures such as a PCDTV on an MDS. The Gen III test phase includes a plan for developing a separation model to ensure the dynamics of this event are better understood to prevent any separation malfunctions. A quantitative understanding of separation dynamics is required to base engineering design decisions on. Previous separation events were based on qualitative assumptions and did not result in favorable test execution results.

\section{Gen I CPAS Boilerplate Drop Test Failure}

The final test of Gen I, CDT-2, was the first conducted using a capsule-shaped vehicle. The Parachute Test Vehicle (PTV) had the same heatshield diameter and lower forebody as the then-current design of the Orion capsule. The PTV was truncated, however, to fit within a cargo aircraft. The PTV was mated with a pallet called the Cradle and Platform Separation System (CPSS) and loaded onto a C-17. An extraction parachute pulled the mated vehicles 
out of the aircraft. Roughly $1.7 \mathrm{~s}$ after extraction, the PTV was released from the CPSS and the parachute sequence began. A programmer parachute and two smaller stabilization parachutes were deployed during separation. The purpose of the stabilization parachutes was to prevent an apex-forward condition immediately after separation,

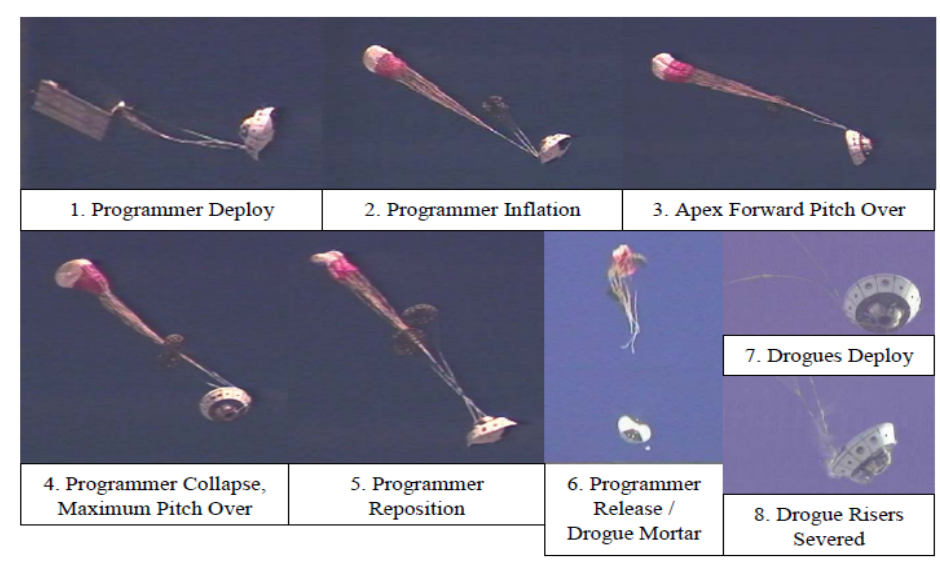

Figure 16. CDT-2 Test Highlights before the programmer was deployed. The programmer parachute was intended to stabilize the PTV. After the programmer and stabilization parachutes were cut away, the full CPAS system was intended to deploy: two mortar-deployed Drogues, three mortardeployed Pilots, and three Pilot-deployed Mains.

In the execution of the test, the programmer failed to control the PTV as shown Figure 16. The vehicle pitched over when the programmer released. Due to the unplanned attitude, the Drogue risers severed on a sharp edge on the vehicle. The PTV fell without parachutes until the centrifugal force pulled the Mains out of their bays. A single damaged Main held onto the vehicle and allowed it to hit bottom-down, the force of the impact caused the vehicle to flip over (Figure 17). The PTV was damaged beyond repair ${ }^{8}$.

The Engineering Investigation Team (EIT) that investigated the CDT-2 failure determined that the primary cause was the inability of the programmer to remain inflated. The EIT specified two detailed causes of the programmer failure, both related to the wake of the test vehicle. First, as on MDT-1, the trailing distance was not appropriately accounted for. Second, the preflight wake analysis had not taken the effect of the stabilization parachutes on the programmer parachute into account. The EIT also identified three contributing factors: the late completion of the concept of operations, the $20 \%$ reefing used on the programmer, and the lack of robust simulations of the extraction and separation phases of flight.

\section{A. Wake Effects}

Preflight analyses were performed to determine the effect of the PTV wake on the programmer parachute. However, two key elements were not taken into account. First, when the PTV pitched over after separation, the programmer riser wrapped around the vehicle. This reduced the trailing distance of the parachute from the expected 5.2 body diameters to a minimum of 2.4 body diameters. The wake in that region caused the programmer to produce less drag than predicted. Like the programmer on MDT-1, it also experienced significant buffeting from the variability in the flow field, further impeding its ability to produce drag.

Preflight analyses did not take into account the effect of the stabilization parachutes on the programmer. Postflight analysis showed that the dynamic pressure at the programmer was reduced to half of the expected value when the stabilization parachutes were included in the model. They also increased the variability in the flow field. The lack of understanding of the wake seen by the programmer parachute was the cause of the programmer failure and thus the failure of the test.

\section{B. Programmer Reefing}

The concept of operations for the separation of the PTV from the CPSS was not finalized until after the vehicles had been built. The attachment point for the programmer that was finally chosen was on the side of the PTV, a point in the structure that had not been designed for parachute loads. To protect the structure, the parachute inflation loads had to be minimized. A $20 \%$ reefing was selected for the first stage of the programmer. However, the reefing line length selected did not take into account the high porosity of the parachute. Post-flight analysis showed that the effective reefing ratio likely was closer to $14 \%$. This was not sufficient for the parachute to stay inflated once the stabilization parachutes were inflated. Both the delay in finalizing the concept of operations and the selection of the small reefing ratio were determined by the EIT to be contributing factors in the test failure. 


\section{Modeling Limitations}

Like the DTV/CMS test configuration, multiple simulations had to be used to simulate the entire trajectory of the PTV and CPSS. DSSA was used to model the extraction and mated flight up to the point of separation. A subcontractor simulated the separation of the PTV and CPSS. Initial conditions from that simulation were input into a version of the Decelerator System Simulation (DSS) specifically modified for this application. The PTV and CPSS were then modeled separately to the ground.

One of the biggest flaws of this simulation architecture was the separation simulation. The separation was only simulated once, very early in the planning of the test. It was not updated based on changes to the configuration. Additionally, only a single set of initial conditions was provided from that simulation to the DSS, rather than a dispersed set. The initial conditions were dispersed in DSS Monte Carlo runs, but the changing configuration forced analysts to use wide dispersions to cover all possible conditions. The high level of uncertainty surrounding the separation of the PTV and CPSS led to the addition of the stabilization parachutes and the use of the programmer attachment point on the side of the vehicle, both of which contributed to the failure as previously described. Had the separation been better understood, these configuration changes likely would not have been implemented, and the test would have succeeded.

\section{Gen I CPAS Boilerplate Failure Recovery}

CPAS formed an Engineering Investigation Team in response to the CDT-2 mishap. This team attributed the test failure to the collapse of the programmer parachute. This programmer collapse was found to be due to insufficient knowledge of the PTV wake environment and inadequate modeling of the separation conditions.9

To address the PTV wake component of the failure, CPAS investigated several test techniques. These included a full-scale wake generating drop test vehicle, and scaled PTV test techniques. Ultimately, the project decided to rely on CFD analysis anchored to wind tunnel testing. CPAS is teaming with the NASA Jet Propulsion Laboratory in planning a wind tunnel test series to gain more knowledge on the wake of the heatshield and its effects on parachute performance.

To improve the understanding of the separation event, CPAS has developed new simulations and new separation techniques. The separation sequence of test CDT-2 and determined that the initial motion was desirable. Shortly after separation, the PTV achieved the desired heatshield forward attitude. Qualitative analysis of the extraction suggested that the desirable separation was a result of the mated vehicle attitude at the separation event. CPAS decided to take steps to ensure that the PTV/CPSS separation was initiated at a similar attitude on future tests. A Smart Release system ${ }^{10}$ was designed and implemented in the Generation II avionics system to initiate release at a specified attitude, (rather than at a specified time). A series of drop tests were conducted to examine the performance of this release system. A software version of the smart release logic was incorporated into the primary aircraft extraction simulation tools to enable analysts to evaluate potential release conditions.

A two-body 6-DOF simulation is currently being developed to model the motion of the PTV2 and CPSS during the separation event. Considerations are also being included to account for dynamics while the two bodies are in close proximity. This simulation will be patched into the existing simulation architecture to create an end-to-end high fidelity simulation of the extraction, separation, and descent of the PTV2/CPSS test architecture. The separation simulation is designed to be initialized with state vectors extracted from the DSSA taken at the time of smart release. The separation simulation output state vector can be passed back to the legacy CPAS trajectory simulations to provide a complete trajectory. It has been designed with Monte Carlo capability so that the variation in potential release conditions can may be assessed. The integrated simulation capability is intended to provide test planners with more sophisticated analyses of the trajectory from extraction through touchdown.

These improvements address the key findings of the failure investigation from CDT-2. They increase the fidelity of test predictions and will be applied to reduce risk in the design of the complicated PTV tests planned for Gen III.

\section{Gen III PCDTV/MDS Vehicle Developments}

CPAS intends to incrementally improve the fidelity of test articles with more representative hardware and deployment methods for the entire parachute sub-system in Gen III testing. Preference is given to testing from the ubiquitous C-130 aircraft using standard LVAD extraction method. The Gen I flight test called CDT-1 was able to include clusters of all three types of CPAS parachutes by mounting a mockup parachute compartment on a Type $\mathrm{V}$ pallet. The test vehicle was stable under the Main parachutes because it was suspended by four harness legs under a confluence fitting. The Orion parachute attachment design has since changed to an Apollo-like design such that the Drogues and Mains converge to the same gusset. This architecture will induce capsule dynamics at

13

American Institute of Aeronautics and Astronautics 


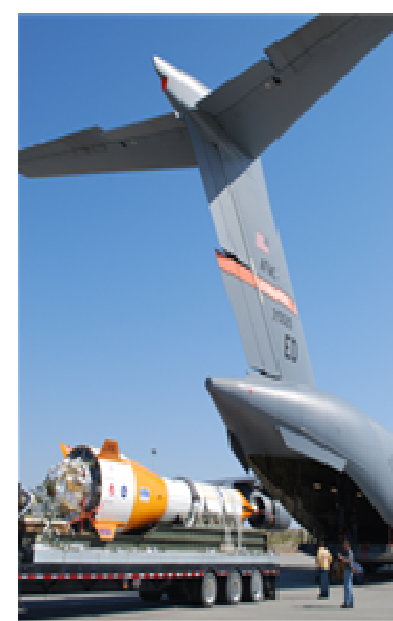

parachute deployment to eventually settle at a static hang angle. Suspending a platform at a single point is not stable and would most likely result in damage upon landing.

There was a strong desire to test the entire landing sub-system with each parachute cluster transmitting loads through production textile and steel risers into the representative Orion attachment structure. Because a platform would be inherently unstable for such a task, CPAS looked towards a dart-shaped test vehicle for this task. The original Solid Rocket Booster parachute test program dropped a missileshaped vehicle from the wing of a B-52 but later employed C-130 aircraft using standard U.S. Army procedures for cost and schedule reasons. ${ }^{11}$ More recently, Alliant Techsystems and United Space Alliance used a missile-shaped Jumbo Drop Test Vehicle (JDTV), or "Jumbo Dart," delivered from a C-17 for the Ares booster parachute flight test program, as shown in Figure $18 .^{12}$

CPAS decided to leverage off Ares experience to design a similar scaled-down system for a C-130. The PCDTV is a missile-shaped test article with a representative parachute compartment attached to the flared aft end. The PCDTV

Figure 18. Loading Ares lies on its side and is secured to a sled called the Mid-Air Delivery System (MDS).
Jumbo Drop Test Vehicle Jumbo Drop Test Vehicle parachute compartment are slightly truncated to fit inside a C-130.

The mated vehicle is extracted from a C-130 with a $28 \mathrm{ft}$ diameter extraction parachute. The MDS repositions to harness legs under the extraction parachute. Immediately afterwards, blankets or straps holding the PCDTV are pyrotechnically severed at a pre-determined time, releasing the PCDTV from the MDS.

DSSA is able to simulate the extraction and reposition of the mated body only until separation. A small modification was made to DSSA to account for having the slings beneath the platform, rather than above the platform as previous tests. The forward momentum of the PCDTV and the deceleration force acting on the MDS ensure positive separation. It was assumed that this separation does not need to be modeled in a standalone simulation because the frictional and aerodynamic interactions between bodies are negligible compared to the virtually instantaneous tendency of the bodies to separate. The state of each body at separation can then be handed off as initial conditions to either DTV-Sim or DSS, depending on the required

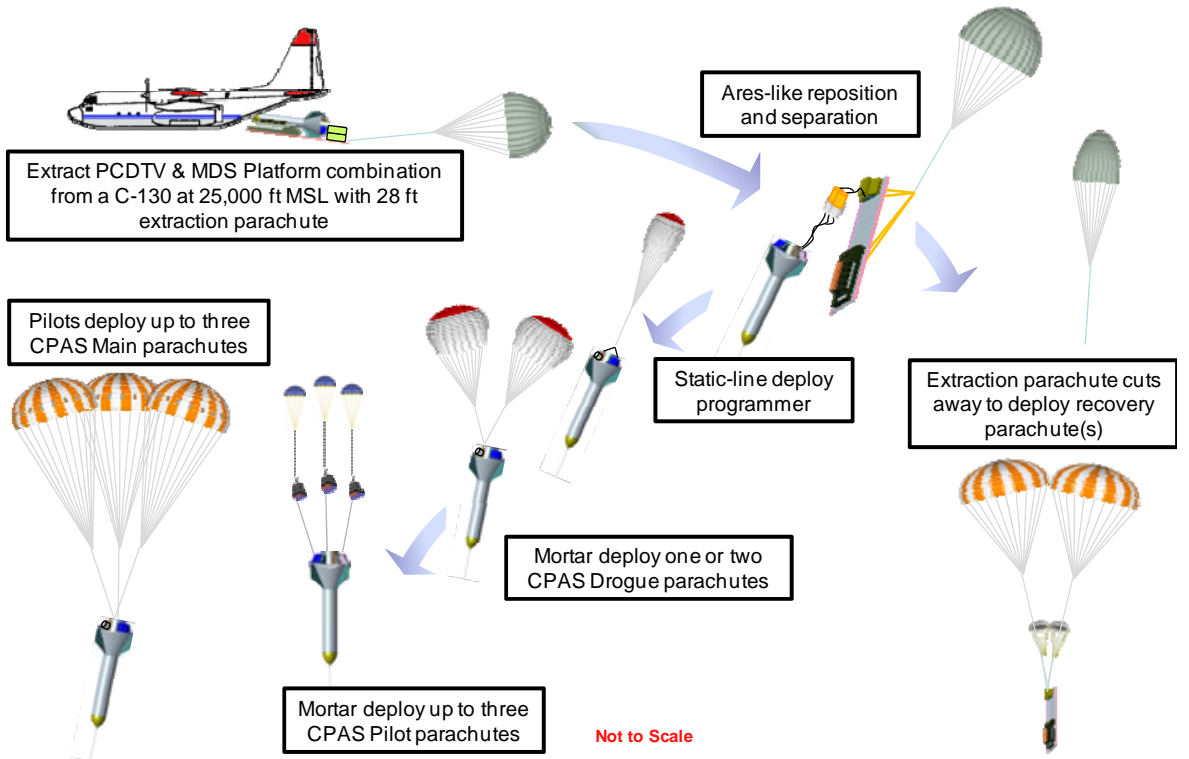
fidelity of simulation.

Upon separation, a programmer is static-line deployed to deliver the PCDTV to the test condition, where the CPAS sequence is activated, beginning with Drogue mortar firing. The MDS will cut away the extraction parachute and descend under recovery parachutes (either G-11s or CPAS Mains). The MDS will land on its front edge, which is covered in honeycomb material to attenuate the shock. 


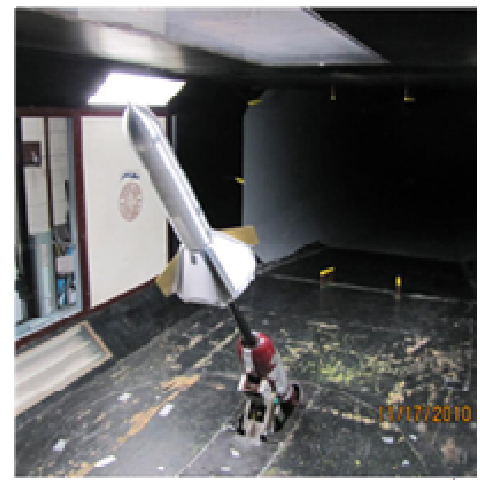

Figure 20. PCDTV wind tunnel test article.

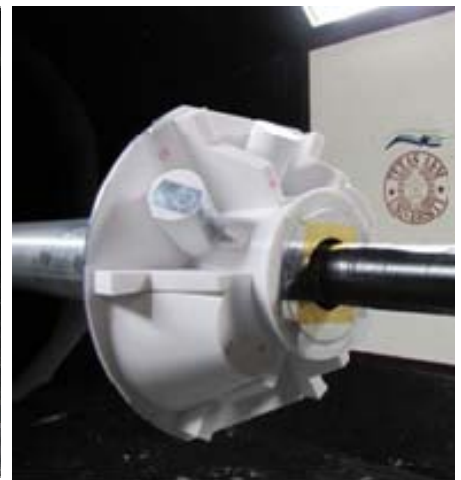

\author{
(1)
}

data, as well as accounting for potential small changes in fin size.

Initial flight test planning and design loads were established with 2-DOF simulations. Improved flight test trajectories and stability assessments will be performed using 6-DOF simulations. Due to its streamlined shape, the PCDTV will not generate an Orion-like wake and the consequential effects of the parachutes.

\title{
X. Gen III CPAS PTV2/CPSS Vehicle Developments
}

Flight testing the PTV2 continues the trend of using hardware more closely representing the final Orion system. A boilerplate vehicle will have more representative vehicle aerodynamics, mass properties, and parachute performance in the presence of an unsteady wake.

The concept of operations and simulation architecture is shown in Figure 21. DSSA will be used to simulate the initial extraction of the mated vehicle from the C-17 up until the simulated "smart release" algorithm commands a separation. The end state from numerous Monte Carlo DSSA trajectories will be used as initial conditions for the custom CPAS Separation Simulation. Each initial condition will be propagated based on the complex interaction of contact forces between the two bodies until the PTV2 is beyond the influence of the CPSS proximity aerodynamics. The states of both articles at that time will be used as initial conditions in DSS for the remainder of each separate simulated trajectory.

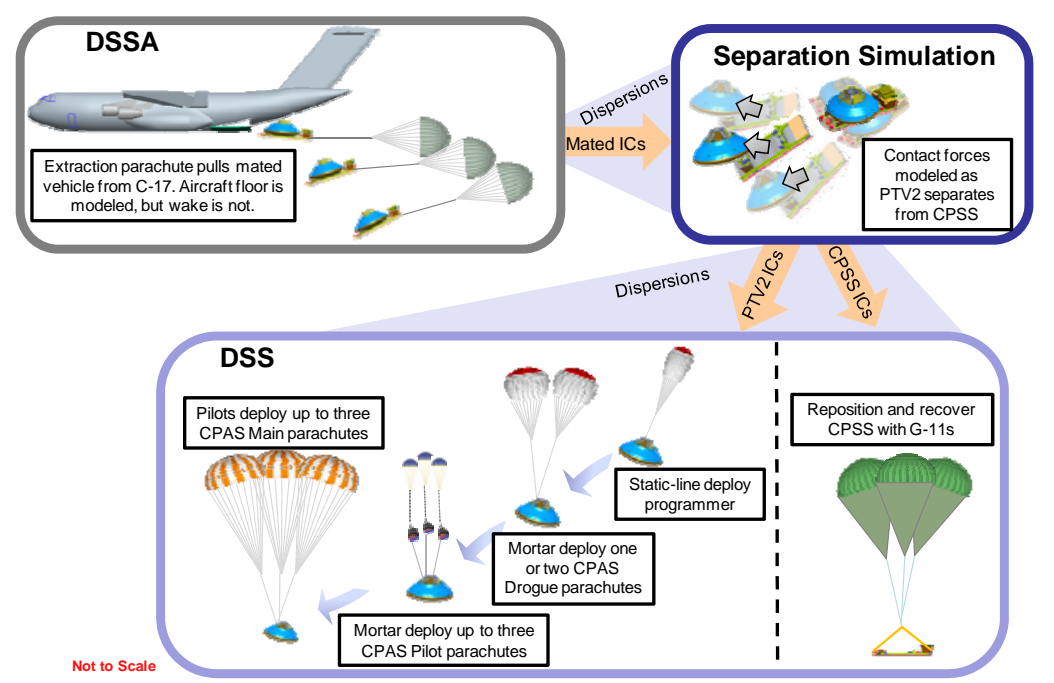

Figure 21. PTV2/CPSS concept of operations and simulation architecture.

The concept of operations adheres to the lessons learned from the CDT-2 recovery plan. The capsule will be released based on the smart release algorithm determined by onboard avionics. Aerodynamic databases of the PTV2 and mated vehicle were established by making small adjustments to previous PTV wind tunnel data to account for the slightly altered outer mold line using CFD. Stabilization parachutes will not be used, to avoid interference with the programmer parachute. Simulations will account for parachute performance degradation in the capsule wake based on aerodynamic wake studies. Below the forward bay, all protuberances with the potential to snatch or sever lines have been eliminated and attachment locations are recessed. 


\section{Gen III PCDTV and PTV2 Heavy Lift Balloon Test Developments}

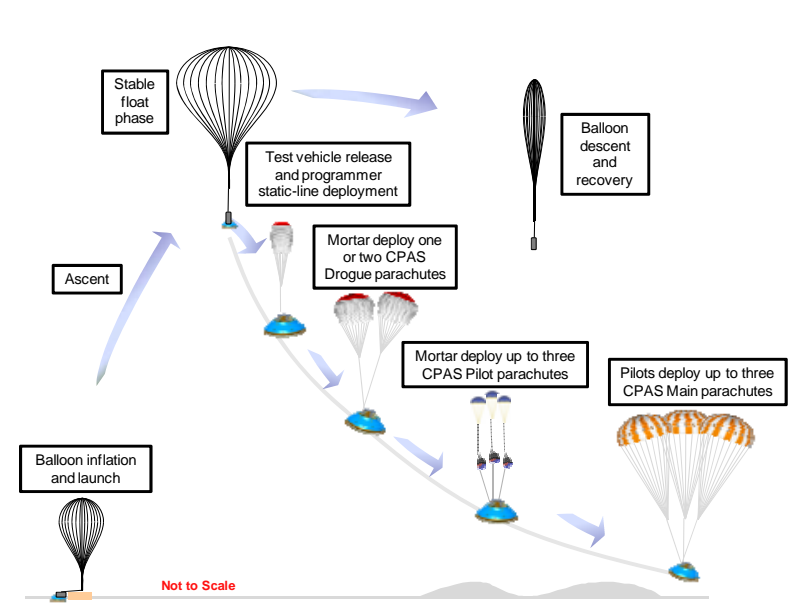

Figure 22. Heavy lift balloon concept of operations for PTV2 or PCDTV. parachute deployment sequence. The balloon will then be deflated significantly for descent and recovery. The size of the programmer and aerodynamics of the test vehicle will determine the airspeed at CPAS Drogue mortar deployment.

Several programs have tested parachutes using balloons deployed from higher altitudes than CPAS is planning including a Mars subsonic parachute ${ }^{13}$ (Jet Propulsion Laboratory and Pioneer Aerospace), the Atmospheric Reentry Demonstrator ${ }^{14}$ (European Space Agency), and the SUPER-M project ${ }^{15,16}$ (Jet Propulsion Laboratory, Wallops Flight Facility, and Near Space Corporation). Because there is no active control of the balloon, a large test range is required. Prevailing winds vary during the year, so testing may be limited by season or multiple test ranges may be necessary for year round testing. During ascent, the balloon will expand to several hundred times its original volume, perhaps over one million cubic meters, which will stress the thin envelope material. The altitude achieved for a given payload weight will be determined by maximizing the amount of expansion and minimizing the weight of the envelope material. Balloon structural failures are common, so the entire payload is often equipped with a large saver parachute. Range considerations will dictate a flight termination system.

\section{Gen I and Gen II Day of Flight Simulations and Lessons Learned}

Safety is the paramount concern of CPAS flight tests. Weather information on the day of flight is used to make several decisions to ensure personnel safety and maximize the potential for test success. Wind measurements from RAWIN balloons are primarily used to compute the footprint of all test articles to ensure landing on the cleared area. The CPAS footprint software, Sasquatch, is constantly undergoing improvement to meet this need, as discussed thoroughly in Ref. ${ }^{17}$. Rather than miss a test date, CPAS will plan for a wind contingency release altitude. In fact, CPAS now routinely runs a family of preflight wind contingency simulations to maximize steady-state time while limiting the footprint. To improve the accuracy of the RAWIN wind measurements, the balloons are released from as close to the drop zone (DZ) as possible.

Altitude data from Gen I and Gen II showed released altitudes were routinely higher than planned by as much as 1,200 feet, reducing the effectiveness of footprint predictions. Because the altitude discrepancy was higher on hotter days, it was discovered to be caused by a miscommunication. Simulations and test planning are based on a geometric release altitude above sea level. However, the flight crew was given this number on the understanding that it was an indicated pressure altitude. Reconstructions of several test day atmospheres showed that these tests were in fact executed at the pressure altitude equal to the desired geometric altitude. Because indicated pressure altitude is based on a standard atmosphere model, this discrepancy was larger on days hotter than a "standard" day. CPAS procedures now include compensating for this effect by providing air crew a target indicated pressure altitude corresponding to the desired geometric altitude based on the day of flight RAWIN atmospheric measurements. Postflight data have shown this new procedure to be effective in consistently matching the planned altitude.

CPAS tests are also limited by surface winds, which might re-inflate parachutes on touchdown and cause a hazard, such as dragging or flipping the test vehicle. Almost all flight tests to date were performed in the desert environment at the U.S. Army Yuma Proving Ground, which has predictable characteristics. Tests are carefully timed to be executed just after sunrise, when winds are a minimum, but ensuring enough sunlight for optical 
tracking. Surface winds are now measured by ground weather stations at both the assembly area and near the center of the DZ to more closely predict the actual winds at touchdown.

\section{Conclusion}

The CPAS project has made significant progress to overcome the numerous challenges related to test and simulation technique developments. Advancements in this area were necessitated to attain a better understanding of parachute performance parameters to ensure an optimum CPAS design is selected for satisfying program requirements essential to qualifying the parachute recovery system. The test and simulation techniques have evolved from using legacy methods to the development of new state-of-the-art techniques that push the envelope of parachute technologies to the next generation.

Early Gen I test efforts involved the use DTVs and Type V LVAD pallet vehicles to test single and cluster parachute configurations. The primary parent aircrafts used to execute CPAS tests were a UH-1, HH-1, and C-130A due to their availability. Test objectives became increasingly demanding and required test conditions to be more representative. A high $\mathrm{q}_{\mathrm{bar}}$ environment was needed to understand inflation performance and resulted in the development of the CMS. The CMS was used to cradle an MDTV onto the cargo area of a C-130A and allowed the MDTV to be released at altitudes not previously attainable by using a helicopter. A PTV and CPSS vehicle was developed in an effort to demonstrate the performance of the parachute system with a representative vehicle. Challenges were experienced during the deployment of this test architecture and the knowledge attained from this experience has contributed to latter test execution methods.

Simulation and analysis techniques used for delivering preflight predictions, mission operation support, and postflight data reconstructions have also evolved to support increasingly complex testing campaigns that involve multiple vehicle systems, separation events, and higher release altitude demands. Early Gen I simulation techniques involved the application of 2-DOF calculations to provide rough order of magnitude analysis. Previous footprint analysis limitations was overcome by the development of Sasquatch. Simulations tools such as DSS, DSSA, and DTV-SIM were introduced and applied to deliver more sophisticated preflight and post-flight analysis. The acquisition of test data and parachute inflation knowledge led to the release of updated versions of each simulation used by CPAS. New analysis techniques were implemented to provide end-to-end trajectory analysis for MDTV/CMS test architectures and Monte Carlo capabilities were extended to all simulations during Gen II. DSS has become the primary simulation for executing post-flight data reconstructions and was adopted for the development of the, "CPAS Engineering Development Unit Operating Modeling Parameters Version 6," and all subsequent parachute modeling memos.

As the project moves forward to Gen III new test and simulations techniques are required to support increasingly complex test concept and vehicle design developments such as the PCDTV and MDS. Limitations in the test and simulation techniques have been identified during Gen I and II. A separation model is planned to be developed during Gen III to ensure decisions are based on mathematical models versus qualitative assumptions to ensure the latest test techniques build on knowledge obtained in Gen I and II. The concurrent evolution of test and simulation techniques has accelerated the project's understanding of parachute performance parameters and prepared the team to meet the demands of the third generation of testing.

\section{Acknowledgments}

The authors wish to thank Peter Cuthbert of NASA-JSC for his contributions to developing the simulations we use today. The simulation advancements made since Gen I would not have been possible without him. Peter Schulte of the University of Texas-Austin and Stephanie Shelton of the University of Alabama-Huntsville also provided a much appreciated review of this paper 


\section{References}

${ }^{1}$ Ray, E., "Challenges of CPAS Flight Testing," 21th AIAA Aerodynamic Decelerator Systems Technology Conference and Seminar, Dublin, Ireland, May 2011 (submitted for publication).

${ }^{2}$ Moore, J., "Development of Monte Carlo Capability for Orion Parachute Simulations," $21^{\text {th }}$ AIAA Aerodynamic Decelerator Systems Technology Conference and Seminar, Dublin, Ireland, May 2011 (submitted for publication).

${ }^{3}$ Cuthbert, P.A. and Conley, G.L., “A Desktop Application to Simulate Cargo Drop Tests," 23- 26 May,2005. 18th AIAA Aerodynamic Decelerator Systems Technology Conference and Seminar, Munich, Germany.

${ }^{4}$ Usbaldo, F.J., Dearman, J., Morris, A., "Proposed Framework for Determining Added Mass of Orion Drogue Parachutes," $21^{\text {th }}$ AIAA Aerodynamic Decelerator Systems Technology Conference and Seminar, Dublin, Ireland, May 2011 (submitted for publication).

${ }^{5}$ Cuthbert, P.A. and Desabrais, K.J., "Validation of a Cargo Airdrop Software Simulator," 19-22 May 2003. 17th AIAA Aerodynamic Decelerator Systems Technology Conference and Seminar, Monterey, California.

${ }^{6}$ Cuthbert, P. (2010) Linear Curve Fit: Gain Schedule For DSS Added Mass. Anaysis Summit v7.

7 “Cradle Monorail System Development for CPAS,” SNELL \& WILMER L.L.P., 20090302164, March 24, 2011, URL: http://www.faqs.org/patents/app/20090302164

${ }^{8}$ Machin, R.A. and Evans, C.T., "Cluster Development Test 2 an Assessment of a Failed Test," May 4-7, 2009. 20th AIAA Aerodynamic Decelerator Systems Technology Conference and Seminar, Seattle, Washington.

${ }^{9}$ Machin, R.A. and Evans, C.T., "Cluster Development Test 2 an Assessment of a Failed Test," May 4-7, 2009. 20th AIAA Aerodynamic Decelerator Systems Technology Conference and Seminar, Seattle, Washington.

${ }^{10}$ Moore, J.W., "Developmetn of a Smart Release Algorithm for Mid-Air Separation of Parachute Test Articles," May 23 - 26, 2011. 21 $1^{\text {st }}$ AIAA Aerodynamic Decelerator Systems Technology Conference and Seminar, Dublin, Ireland. (submitted for publication).

${ }^{11}$ Runkle, R. E. and Wolf, D. F., "Space Shuttle Solid Rocket Booster Lightweight Recovery System," $13^{\text {th }}$ AIAA Aerodynamic Decelerator Systems Technology Conference, Clearwater Beach, FL, May 15-18, 1995, AIAA paper 1995-1594.

12“'Ares Parachute Drop Test in Yuma,” Constellation Multimedia Gallery, October 8, 2009, accessed March 4, 2011, URL: http://www.nasa.gov/mission_pages/constellation/multimedia/ares/droptest_oct09.html

${ }^{13}$ Mitcheltree, R., Bruno, R., Slimko, E., Baffes, C., Konefat, E., and Witkowski, A., "High Altitude Test Program for a Mars Subsonic Parachute," $18^{\text {th }}$ AIAA Aerodynamic Decelerator Systems Technology Conference and Seminar, Munich, Germany, May 23-26, 2005, AIAA paper 2005-1659.

14 Rives, J. and Leveugle, T., "Atmospheric Re-entry Demonstrator Descent and Recovery Sub-System Qualification Test Capsule Stabilization during the Free Fall Phase," $14^{\text {th }}$ AIAA Aerodynamic Decelerator Systems Technology Conference, San Francisco, CA, June 3-5, 1997, AIAA paper 1997-1440.

${ }^{15}$ Hall, J. L., et al., "Flight Test Results for Aerially Deployed Mars Balloons," AIAA Balloon Systems Conference, Williamsburg, VA, May 21-24, 2007, AIAA paper 2007-2626.

${ }^{16}$ Hall, J. L., et al., "Mars Balloon Flight Test Results," AIAA Balloon Systems Conference, Seattle, WA, May 4-7, 2009, AIAA paper 2009-2809.

17 Bledsoe, K., "Development of the Sasquatch Drop Test Footprint Tool," May 23 - 26, 2011. 21 $1^{\text {st }}$ AIAA Aerodynamic Decelerator Systems Technology Conference and Seminar, Dublin, Ireland. (submitted for publication). 